\title{
What's the score? Adaptation of probability of adequate nutrient intake scores to the National Adult Nutrition Survey database using European reference values
}

\author{
L.B. Kirwan ${ }^{1}$, A. Nugent ${ }^{1,2}$, J. Walton ${ }^{3}$, A. Flynn ${ }^{4}$ and B.A. McNulty ${ }^{1}$ \\ ${ }^{1}$ UCD Institute of Food and Health, University College Dublin, Dublin, Ireland, \\ ${ }^{2}$ Institute for Global Food Security, School of Biological Sciences, Queens University Belfast, UK, \\ ${ }^{3}$ Department of Biological Sciences, Munster Technological University, Cork, Ireland and \\ ${ }^{4}$ School of Food and Nutritional Sciences, University College Cork, Cork, Ireland
}

Nutrient-based diet quality scores provide an objective approach to diet quality and are adaptable to different populations and countries. Nutrient scores have been used in inter-disciplinary research in recent years ${ }^{(1)}$. Probability of adequate nutrient diet (PANDiet) scores are assigned to individuals in a food consumption database and range from 0 to 100; with a higher score indicating better diet quality $^{(2)}$. This score covers 24 nutrients and has been validated on databases in France, Germany, Italy, the UK, and the US on various population groups ${ }^{(2-4)}$. As converting detailed nutrient intake into a single score can result in a loss of transparency and interpretability, it is imperative that nutrient scores are comprehensive and validated before use ${ }^{(2)}$.

The aim of the current study was to assign PANDiet scores to National Adult Nutrition Survey food consumption data (NANS, 2008-2010, www.iuna.net). Scores were assigned according to a protocol defined for French and US databases ${ }^{(2)}$ using RStudio version 3.5.2 statistical software and IBM SPSS Statistics version 26.0. As cholesterol intakes were unavailable, PANDiet scores were calculated on the intakes of 23 nutrients ( 6 macronutrients, 10 micronutrients and 7 minerals). A penalty score of 0 was assigned to intakes exceeding the upper limit for 17 nutrients and European nutrient reference values were used as cut off points.

PANDiet scores aligned with previous studies and were correlated at a low level with energy intake $(\mathrm{r}=0.11, \mathrm{P}<0.0001)$. The mean PANDiet score was similar to that calculated for French and US databases (64.95 \pm 0.24 , range 45.05-92.99). Correlation between PANDiet scores and nutrient sub-scores were also as expected, apart from vitamin B12 which had a significant positive relationship in NANS $(r=0.41, p<0.01)$. A higher PANDiet score was associated with higher intakes of breakfast cereals, breads, fruit, vegetables, yoghurt, reduced fat milks, unprocessed white meat and lower intakes of fats and spreads, cheese and processed red meat. Participants with a higher PANDiet score were more likely to be female $(p=0.0004)$, older $(p=0.003)$, non-smokers and have low energy dense diets (both $\mathrm{p}<0.0001$ ) after controlling for appropriate factors. Higher PANDiet scores were correlated to lower urinary sodium levels and higher plasma folate and riboflavin status (all $\mathrm{p}<0.0001)$.

The PANDiet scoring system provides a useful metric for nutrient adequacy and dietary quality at an individual and population level. Comprehensive, robust and validated nutrient scoring systems may prove a valuable metric for inter-disciplinary research in the future and facilitate a cross-country comparison of diets.

\section{Acknowledgements}

The National Adult Nutrition Survey was funded by the Irish Department of Agriculture, Food and the Marine (DAFM).

\section{References}

1. Seconda L, Baudry J, Pointereau P, et al. (2019) Br J Nutr 121(10), 1166-1177

2. Verger EO, Mariotti F, Holmes BA, et al. (2012) PLoS One 7(8)

3. Schoen S, Jergens S, Barbaresko J, et al. (2017) Nutrients 9(1)

4. Mistura L, D’Addezio L, Sette S, et al. (2016) Int J Food Sci Nutr 67(3), 232-238 